\title{
Correction: Interleukin-1 receptor on hippocampal neurons drives social withdrawal and cognitive deficits after chronic social stress
}

Damon J. DiSabato - Daniel P. Nemeth - Xiaoyu Liu • Kristina G. Witcher - Shane M. O'Neil (D) Braedan Oliver • Chelsea E. Bray · John F. Sheridan (1) · Jonathan P. Godbout • Ning Quan (i)

Published online: 30 June 2020

(c) Springer Nature Limited 2020

Correction to: Molecular Psychiatry

https://doi.org/10.1038/s41380-020-0788-3

published online 22 May 2020

Following publication of this article the authors noticed that the GEO series accession number was accidentally omitted. The following data availability statement has now been included in the article:
"The RNA-sequencing data discussed in this publication have been deposited in NCBI's Gene Expression Omnibus (DiSabato et al., 2020) and are accessible through GEO Series accession number GSE149195 (https://www.ncbi. nlm.nih.gov/geo/query/acc.cgi?acc=GSE149195)."

This has been corrected in both the PDF and HTML versions of the article. 